\title{
Predictive value of chest computed tomography for axillary lymph node metastasis in patients with breast cancer A retrospective cohort study
}

\section{Chohee Kim, Myung Jin Chung, Semin Chong}

Department of Radiology, Samsung Medical Center, Sungkyunkwan University School of Medicine, Seoul, Korea

Received: July 2, 2021

Revised: July 6, 2021

Accepted: July 7, 2021

Corresponding author:

Semin Chong

Department of Radiology, Samsung Medical Center, Sungkyunkwan University School of Medicine, 81 Irwon-ro, Gangnam-gu, Seoul 06351, Korea Tel: +82-2-3410-2518

E-mail:sm.chong@samsung.com

\begin{abstract}
Purpose: This study aimed to evaluate the predictive value of preoperative chest computed tomography (CT) for axillary lymph node (ALN) metastasis in patients with breast cancer.

Methods: CT features of ALNs were retrospectively reviewed in 212 patients with breast cancer who underwent preoperative chest CT examination and ALN dissection. Primary tumor size and CT characteristics of ALNs (cortical thickness, cortical shape, the presence or absence of contrast enhancement of ALNs, and the presence or absence of perinodal infiltration) were recorded and analyzed. A nomogram was developed to visualize the associations between the predictors and each ALN status endpoint.

Results: Of 212 patients, 95 (44.8\%) had ALN metastasis. Primary tumor size and CT characteristics of ALNs were identified as predictors of ALN metastasis. The nomogram comprising primary tumor size and cortical shape was a good validation model for predicting ALN metastasis. The sensitivity, specificity, and accuracy of the nomogram for predicting ALN metastasis were $88.4 \%, 79.5 \%$, and $83.5 \%$, respectively.

Conclusion: Using preoperative chest CT scans, a nomogram combining the cortical shape of ALNs with the primary tumor size showed good performance in predicting ALN metastasis.
\end{abstract}

Keywords: Axilla; Breast neoplasms; Lymph nodes; Lymphatic metastasis; Tomography, X-ray computed

\section{INTRODUCTION}

Axillary lymph node (ALN) metastasis has traditionally been considered as the most important prognostic factor in breast cancer [1]. Preoperative evaluation of ALN metastasis in patients with breast cancer has been mainly performed using breast or axillary ultrasound (US), chest computed tomography (CT), or breast magnetic resonance imaging (MRI), and its need is increasing because ALN dissection (ALND) is replaced by sentinel lymph node (SLN) biopsy in
This is an Open Access article distributed under the terms of the Creative Commons Attribution Non-Commercial License (https:// creativecommons.org/licenses/ by-nc/4.0/). 
clinically ALN-negative patients [2]. Recent clinical trials reported that ALND was unnecessary even if 1-2 SLN metastases were detected [3-6]. Therefore, it is essential to evaluate ALN metastasis before treatment or surgery for making treatment decisions and establishing the postoperative status in patients with breast cancer.

Preoperative axillary US has become a routine practice to predict the axillary nodal status in combination with core needle biopsy or fine-needle aspiration cytology for breast cancer work-up in many institutions [7]. However, this procedure has limitations in that it can lead to an operator-dependent manner with a wide range of sensitivity (50\% to 92\%) and potentially implantation metastasis [7]. In addition, preoperative breast MRI of patients with breast cancer has been evaluated for ALN metastasis; however, inconsistent results have been reported, which are controversial in studies of MRI performance for evaluating ALN metastasis [8-10].

As $\mathrm{CT}$ is the primary method for evaluating the regional lymph node status before surgery in many other cancer staging systems [11-13], clinicians are increasingly using chest CT to assess locoregional and pulmonary metastasis in patients with breast cancer preoperatively. Previous chest CT studies reported that metastatic ALNs were characterized by smaller diameters of 5 to $10 \mathrm{~mm}$ or more, lack of fat density in the node, circular or irregular morphology, and early contrast enhancement [14-16]. However, it is not easy to evaluate fatty hilum, enhancement, and shape accurately and consistently in relatively small ALNs. In this study, we devised a predictive model for ALN metastasis with the primary tumor size, focusing on the cortical CT morphology and perinodal invasion of ALNs, which can reflect tumor cell deposition and local invasion of pathologic lymph nodes. Therefore, this study aimed to evaluate the morphology of ALNs on a preop- erative chest CT scan and to analyze its ability to predict ALN metastasis in patients with breast cancer.

\section{METHODS}

\section{Patient enrollment}

This retrospective study was approved by the Institutional Review Board (2020-09-219-001) of Samsung Medical Center. The requirement for informed consent was waived. Between January 2012 and December 2013, patients with primary breast cancer who underwent preoperative chest CT with both pre- and post-contrast enhancement protocols and subsequent surgery for axillary staging, including ALND, were initially considered eligible for this study $(n=353)$, and their electronic medical records were reviewed. Patients were excluded according to the following conditions. (1) Pre- or postcontrast enhanced chest CT scans of 51 patients had a poor image quality. (2) Eighteen patients presented with bilateral breast cancers with a history of previous cancer treatment including neoadjuvant chemotherapy or radiation therapy. (3) Sixty-five patients underwent fine-needle aspiration or core needle biopsy for ALNs prior to CT scans within 7 days due to post procedure related change. (4) Seven patients had incomplete medical records. Finally, a total of 212 patients with a power of 0.95 at a significance level of 0.05 and an effect size of 0.5 were selected (Fig. 1 ).

\section{Scan protocol of chest CT}

All patients underwent examination with a 64-detector CT scanner (LightSpeed VCT 64, GE Healthcare, Milwaukee, WI, USA), with the following parameters: a voltage of $120 \mathrm{kV}$, automatic tube current modulation (100 to $120 \mathrm{mAs}$ ), $0.984 \times$ $1.375 \mathrm{~mm}$ pitch, $2 \mathrm{~mm}$ slice thickness, table speed of 175

From January 2012 to December 2013, 353 patients with primary breast cancer and undergone preoperative chest $\mathrm{CT}$ in our institution

141 Patients excluded due to:

51 Patients without proper chest CT

18 Patients with bilateral breast cancer or history of previous cancer treatment 65 Patients with recent fine needle aspiration of axillary lymph nodes

7 Patients with insufficient medical records

212 Cases were enrolled in the study

Fig. 1. Flowchart of selecting patients with breast cancer. CT, computed tomography. 
$\mathrm{mm} / \mathrm{sec}, 2.5$ rotation/sec, a matrix size of $512 \times 512$, and CT dose reduction protocol with adaptive statistical iterative reconstruction. All chest CT examinations were performed on the patients from the level of the lower neck to the bottom of the thorax in the supine position with bilateral arms over their heads. Each scan was acquired during a single breath-hold. The coronal image was reconstructed at $2 \mathrm{~mm}$ intervals from the coronal plane of the axial image.

\section{CT evaluation of the ALNs}

The two sets of the axial and coronal images were evaluated using a picture archiving and communicating system in a mediastinal window setting to a window level of 50 and a window width of 350. Preoperative chest CT images of each patient were reviewed retrospectively by consensus between two thoracic radiologists, each having an experience of 16 and 2 years, respectively.

The largest ALN on the affected side was selected on the chest CT image. The recorded parameters were as follows: (1) cortical thickness (defined thickest diameter of the short axis of the cortical region of the ALN); (2) cortical shape (I, thin curvilinear; II, diffuse thickened curvilinear $>3 \mathrm{~mm}$; III, nodular thickened; IV, rounded or irregular) (Fig. 2); (3) the presence or absence of strong contrast enhancement compared to the attenuation of the pectoralis major muscle; (4) the presence or absence of perinodal infiltration according to the clarity of the boundary between ALN and axillary fat. The primary tumor stage $(\mathrm{T})$ was recorded as Tis (carcinoma in situ), $\mathrm{T} 1$ ( $\leq 20 \mathrm{~mm}$ ), T2 (>20 $\mathrm{mm}$ but $\leq 50 \mathrm{~mm}$ ), T3 (>50 mm) and T4 (any size growing into the chest wall or skin), along with the primary tumor type and the presence or absence of ALN metastasis according to the postoperative pathologic report.

\section{Statistical analysis}

All 212 patients were analyzed using binary subgroup analysis of non-ALN metastasis versus ALN metastasis. Differences in categorical variables were tested using the chi-square test or Fisher's exact test. Logistic regression analysis was performed to identify the independent factors associated with ALN metastasis among the variables. Multivariate analysis was used to account for multicollinearity between the variables. In each case, the model including only significant variables was estimated using the backward selection method and validated for the model's fitness by R-square, max-rescaled R-square, Hosmer-Lemeshow (HL) goodness-of-fit test, Akaike information criterion (AIC), and likelihood ratio statistics (LRS). Results are presented as odds ratios (ORs) with 95\% confidence intervals. The regression coefficients estimated from the multivariate logistic regression models were illustrated graphically in nomograms, which provided a straightforward method for predicting the extent of axillary disease. The receiver operating characteristic (ROC) curve was drawn, and the area under the curve (AUC) was used to assess the predictive accuracy of the model. The optimal cutoff value was based on the ROC curve with Youden's J statistic (J), J=sensitivity + specificity-1. Differences were considered statistically significant at $P<0.05$. Statistical analysis was performed using the SAS software version 9.4 (SAS Institute Inc., Cary, NC, USA) and R Project version 3.4.3 (R Foundation for Statistical Computing, Vienna, Austria; http://www. r-project.org/).

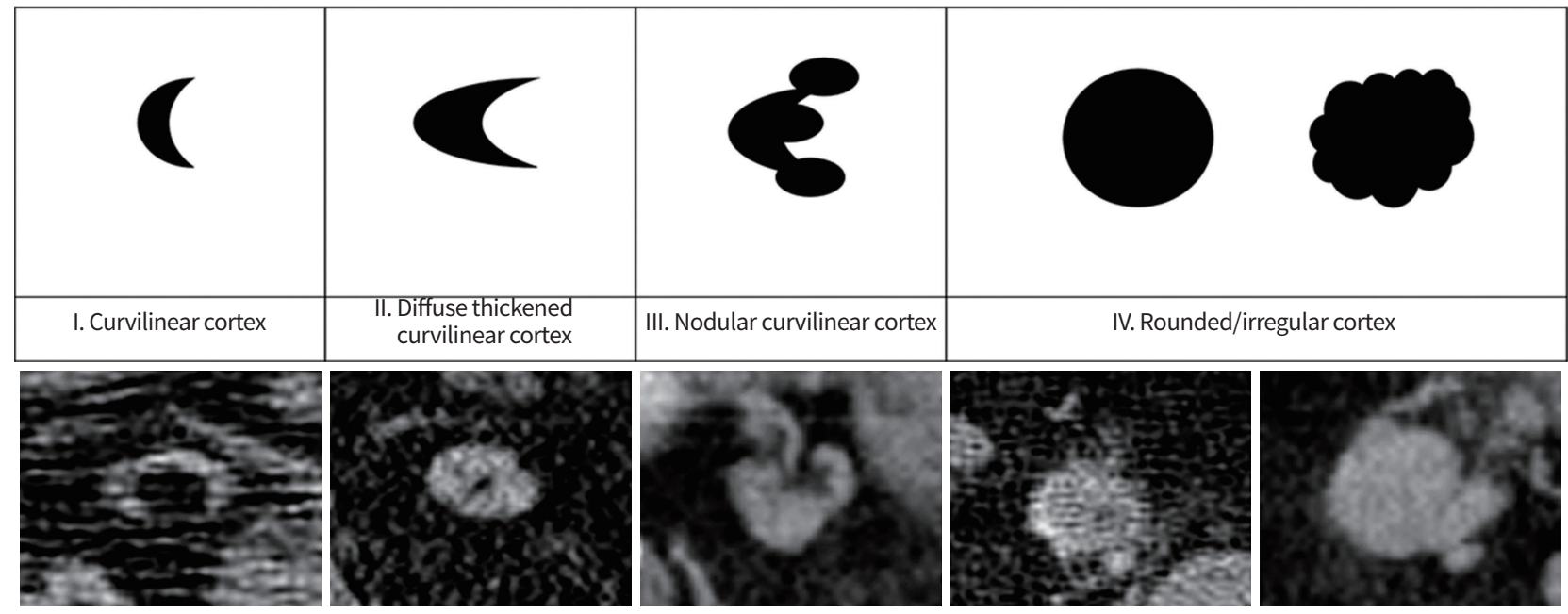

Fig. 2. Schematic illustration of the cortical shape of the axillary lymph node on chest computed tomography. (I) Thin curvilinear cortex. (II) Diffuse thickened curvilinear more than $3 \mathrm{~mm}$. (III) Nodular thickened cortex. (IV) Rounded/irregular cortex. 


\section{RESULTS}

\section{Subject characteristics}

All 212 patients were females, with an average age of 50 years (range, 23 to 78). The primary tumor size was Tis and $\mathrm{T} 1$ in 95 (44.8\%) patients, T2 in 98 (46.2\%) patients, and more than T3

Table 1. Demographics of 212 breast cancer patients with or without axillary lymph node metastasis

\begin{tabular}{|c|c|c|c|c|}
\hline \multirow{2}{*}{ Demographic } & \multirow{2}{*}{$\begin{array}{c}\text { All } \\
\text { patients }\end{array}$} & \multicolumn{2}{|c|}{ ALN metastasis } & \multirow{2}{*}{ P-value } \\
\hline & & Negative & Positive & \\
\hline Female sex & 212 & $117(55)$ & $95(45)$ & \\
\hline Age (yr) & $50(23-78)$ & $49(23-78)$ & $50(28-74)$ & 0.6283 \\
\hline \multicolumn{5}{|l|}{ Primary tumor site } \\
\hline Right & $88(42)$ & $52(44)$ & $36(38)$ & 0.3358 \\
\hline Left & $124(58)$ & $65(56)$ & $59(62)$ & \\
\hline T stage & & & & $<0.0001$ \\
\hline Tis and T1 (ref) & 95 & $69(59)$ & $26(27)$ & \\
\hline $\mathrm{T} 2$ & 98 & $46(39)$ & $52(55)$ & 0.0003 \\
\hline $\mathrm{T} 3$ and T4 & 19 & $2(2)$ & $17(18)$ & $<0.0001$ \\
\hline
\end{tabular}

Values are presented as number (\%) or median (range). All except age values are the number of breast cancer patients.

ALN, axillary lymph nodes. in 19 (9.0\%) patients. T stage of the primary tumor was significantly higher in breast cancers with ALN metastasis $(P<0.001)$. The primary tumor types were invasive breast carcinoma in 199 (93.9\%) patients and ductal carcinoma in situ in 13 (6.1\%) patients. Among the 212 patients, 95 (44.81\%) patients had ALN metastasis, and 117 (55.19\%) patients did not have ALN metastasis (Table 1).

\section{CT characteristics of ALNs}

Table 2 shows the CT comparison of ALNs with and without metastasis. The cortical thickness of metastatic ALNs was significantly thicker than that in non-metastatic ALNs (3.68 \pm $1.57 \mathrm{~mm}$ vs. $7.20 \pm 5.26 \mathrm{~mm}, \mathrm{P}<0.0001$ ) (Figs. 3-5). Nodular curvilinear cortical shape $(P<0.001 ; O R, 26.31)$ and round/ irregular cortical shape $(P<0.001 ; O R, 102.0)$ of ALNs were significantly associated with ALN metastasis (Figs. 4, 5). The presence of strong contrast enhancement $(P<0.001$; OR, 5.04) of ALNs and perinodal infiltration of ALNs ( $P<0.001$; OR, 18.37) were also significant CT predictors of ALN metastasis.

\section{Univariate and multivariate analysis}

Table 2 shows the univariate analysis of the primary tumor size, cortical thickness, cortical shape, strong contrast enhancement, and perinodal infiltration of ALN, which were significantly associated with ALN metastasis. In the validation of

Table 2. Comparison of computed tomography characteristics of axillary lymph node metastasis

\begin{tabular}{|c|c|c|c|c|c|}
\hline \multirow{2}{*}{ Characteristic } & \multirow{2}{*}{ All patients } & \multicolumn{2}{|c|}{ ALN metastasis } & \multirow{2}{*}{ P-value } & \multirow{2}{*}{ OR $(95 \% \mathrm{CI})$} \\
\hline & & Negative & Positive & & \\
\hline Cortical thickness (mm) & $5.26 \pm 4.09$ & $3.68 \pm 1.57$ & $7.20 \pm 5.26$ & $<0.0001$ & $8.53(4.19-17.36)$ \\
\hline Cortical shape $^{a)}$ & & & & $<0.0001$ & \\
\hline I (ref) & 56 & $51(44)$ & $5(5)$ & & 1 \\
\hline ॥ & 55 & $44(38)$ & $11(12)$ & 0.1049 & $2.55(0.82-7.91)$ \\
\hline III & 68 & $19(16)$ & $49(52)$ & $<0.0001$ & $26.31(9.11-75.95)$ \\
\hline IV & 33 & $3(3)$ & $30(32)$ & $<0.0001$ & $102.00(22.74-457.44)$ \\
\hline \multicolumn{6}{|l|}{ Contrast enhancement } \\
\hline Not prominent & 105 & $78(67)$ & $27(28)$ & $<0.0001$ & \\
\hline Prominent & 107 & $39(33)$ & $68(72)$ & & $5.04(2.80-9.08)$ \\
\hline \multicolumn{6}{|l|}{ Perinodal infiltration } \\
\hline Absent & 187 & $115(98)$ & $72(76)$ & $<0.0001$ & \\
\hline Present & 25 & $2(2)$ & $23(24)$ & & $18.37(4.20-80.25)$ \\
\hline
\end{tabular}

Values are presented as mean \pm standard deviation or number (\%). All except cortical thickness values are numbers of breast cancer patients. ALN, axillary lymph nodes; $\mathrm{OR}$, odds ratio; $\mathrm{Cl}$, confidence interval.

${ }^{a)}$ Cortical shape of ALN was categorized. (I) Thin curvilinear cortex. (II) Diffuse thickened curvilinear more than 3 mm. (III) Nodular thickened cortex. (IV) Rounded/irregular cortex. 

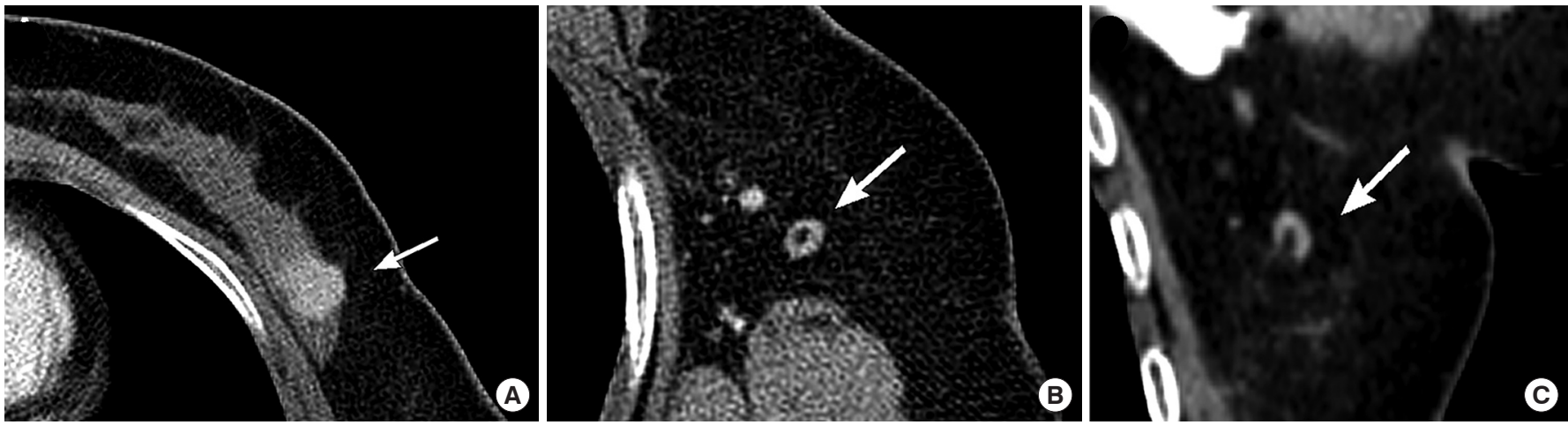

Fig. 3. Postcontrast chest computed tomography (CT) images of a 56-year-old woman with stage IA breast cancer (pT1pN0). (A) Axial CT image shows a 15-mm-sized primary tumor in the left breast (arrow). Axial (B) and coronal (C) CT images show a normal left level I axillary lymph node with the thin cortex (type I) (arrows). The cortical thickness was measured to be $2.7 \mathrm{~mm}$. There is no perinodal infiltration and no strong enhancement.
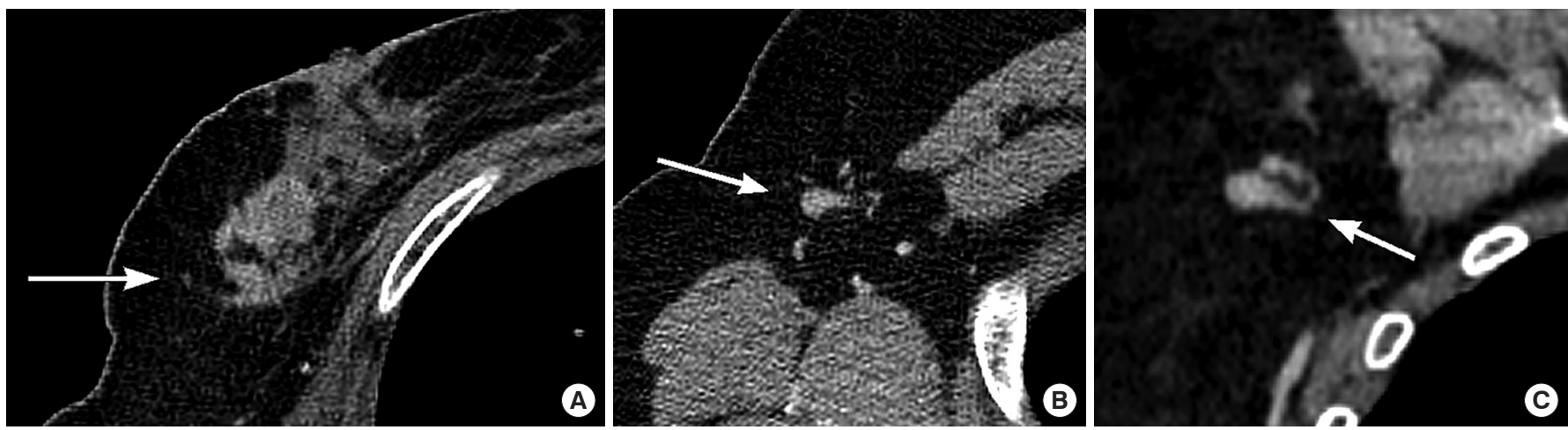

Fig. 4. Postcontrast chest computed tomography (CT) images of a 59-year-old woman with stage IIIA breast cancer (pT3pN1). (A) Axial CT image shows a clinical T3 primary tumor in the right breast but not fully covered (arrow). Axial (B) and coronal (C) CT images show a right level I axillary lymph node with the nodular curvilinear cortex (type III), which is suggestive of metastatic lymphadenopathy (arrows). The cortical thickness was measured to be $6.3 \mathrm{~mm}$.
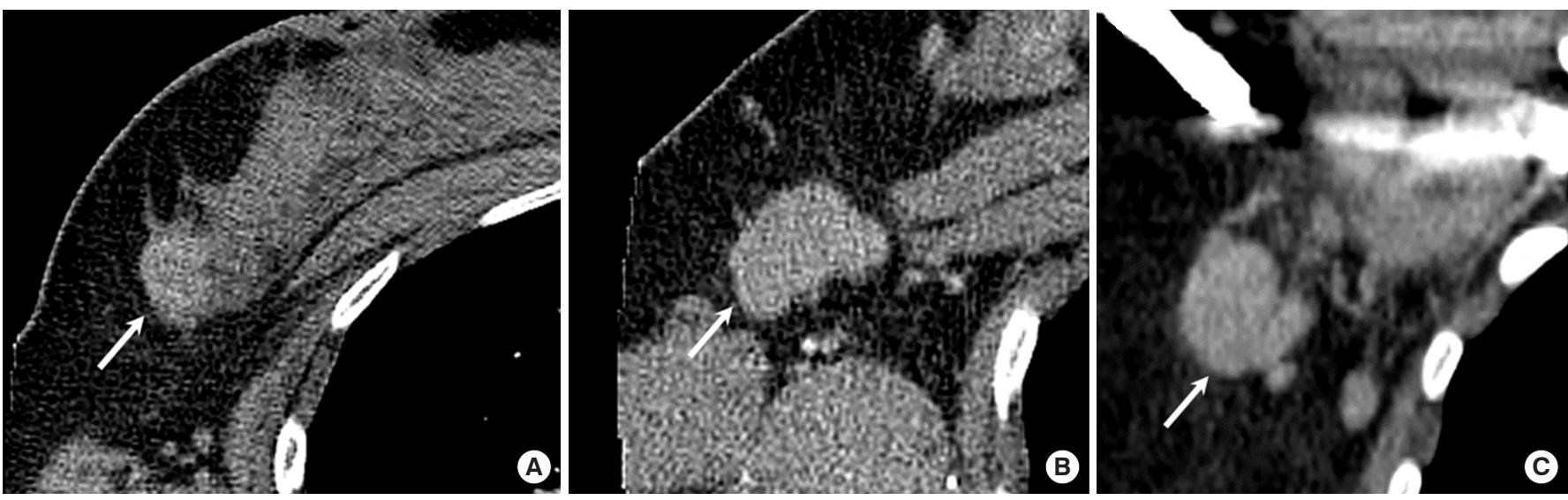

Fig. 5. Postcontrast chest computed tomography (CT) images of a 49-year-old woman with stage IIIB breast cancer (pT2pN3). (A) Axial CT image shows a clinical T2 primary tumor in the right breast but not fully covered (arrow). Axial (B) and coronal (C) CT images show a right level I axillary lymph node with the irregular cortex (type IV), which is suggestive of metastatic lymphadenopathy (arrows). The cortical thickness was measured to be $16 \mathrm{~mm}$. 
the model's fitness by R-square, max-rescaled R-square, $\mathrm{HL}$ goodness-of-fit test, AIC, and LRS, the model with the cortical shape and T stage was found to be more suitable for predicting ALN metastasis than the model including cortical thickness, enhancement, perinodal infiltration, and T stage. Table

Table 3. Multivariate logistic regression analysis for cortical shape and primary tumor size predicting axillary lymph node metastasis

\begin{tabular}{lcc}
\hline Variable & OR $(95 \% \mathrm{Cl})$ & P-value \\
\hline $\begin{array}{lc}\text { Cortical shape }{ }^{\mathrm{a})} \\
\text { I (ref) }\end{array}$ & 1 & $<0.0001$ \\
II & $1.87(0.53-6.55)$ & 0.3306 \\
III & $28.59(8.95-91.40)$ & $<0.0001$ \\
IV & $92.95(19.16-450.81)$ & $<0.0001$ \\
T stage & & $<0.0010$ \\
Tis and T1 (ref) & 1 & \\
T2 & $2.70(1.19-6.13)$ & 0.0177 \\
T3 and T4 & $38.57(6.54-227.65)$ & $<0.0001$ \\
\hline
\end{tabular}

OR, odds ratio; $\mathrm{Cl}$, confidence interval.

${ }^{a)}$ Cortical shape of axillary lymph node was categorized. (I) Thin curvilinear cortex. (II) Diffuse thickened curvilinear more than $3 \mathrm{~mm}$. (III) Nodular thickened cortex. (IV) Rounded/irregular cortex.
3 shows the multivariate logistic regression analysis of the cortical shape of ALN and the primary tumor size used to construct the nomogram predicting ALN metastasis. The HL goodness-of-fit test resulted in a P-value of 0.8642 , which indicated that the model fit well. The model generated a cutoff value to estimate ALN metastases, which was a score of 74 , and the sensitivity and specificity of the predictive nomogram were calculated for any given cutoff value of the risk estimate score (Fig. 6). A total of $>74$ points using a nomogram including the cortical shape of ALN and primary tumor size showed a sensitivity of $88.4 \%$, specificity of $79.5 \%$, a positive predictive value of $77.8 \%$, a negative predictive value of $89.4 \%$, and an accuracy of $83.5 \%$. The AUC for positive ALN was 0.8915 (Fig. 7).

\section{DISCUSSION}

In our study, a nomogram was devised that combined the cortical CT morphology (cortical shape) and primary tumor size as a predictor of ALN metastasis in breast cancer. Our model demonstrated a sensitivity of $88.4 \%$, specificity of $79.5 \%$, and accuracy of $83.5 \%$. Several previous CT studies reported that the sensitivity and specificity of ALN metastasis

Points

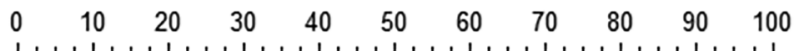

\begin{tabular}{llr}
\hline Shape & Points & \\
1 & 0 \\
& 2 & 14 \\
& 3 & 74 \\
4 & 100
\end{tabular}

Cortical shape

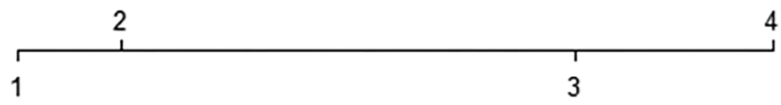

Primary tumor size

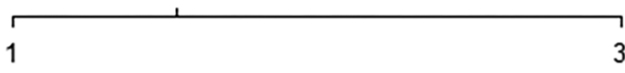

Total points

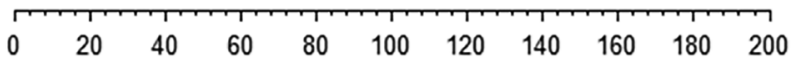

T3

Total points
Points

1 0

22

3

81

$\begin{array}{rr}2 & 0.05 \\ 19 & 0.1 \\ 36 & 0.2 \\ 48 & 0.3 \\ 58 & 0.4 \\ 67 & 0.5 \\ 76 & 0.6 \\ 86 & 0.7 \\ 98 & 0.8 \\ 116 & 0.9 \\ 168 & 0.99\end{array}$

Fig. 6. Nomogram predicting axillary lymph node metastasis in breast cancers. To visualize a multivariate logistic regression model to define axillary lymph node metastasis based on cortical shape and primary tumor size, the nomogram is used by first giving each variable a score on its points scale. Total points are obtained from the scores of all variables, and the probability of the event, i.e., axillary lymph node metastasis, is estimated by drawing a vertical line from the total point. In cortical shape, I, thin curvilinear; Il, diffuse thickened curvilinear more than 3 mm; III, nodular thickened; IV, rounded or irregular. In primary tumor size, $1, \leq 20 \mathrm{~mm} ; 2,>20$ and $\leq 50 \mathrm{~mm} ; 3, \geq 50 \mathrm{~mm}$. 
PRECISION AND FUTURE MIEDICINE

CT prediction of axillary lymph node metastasis
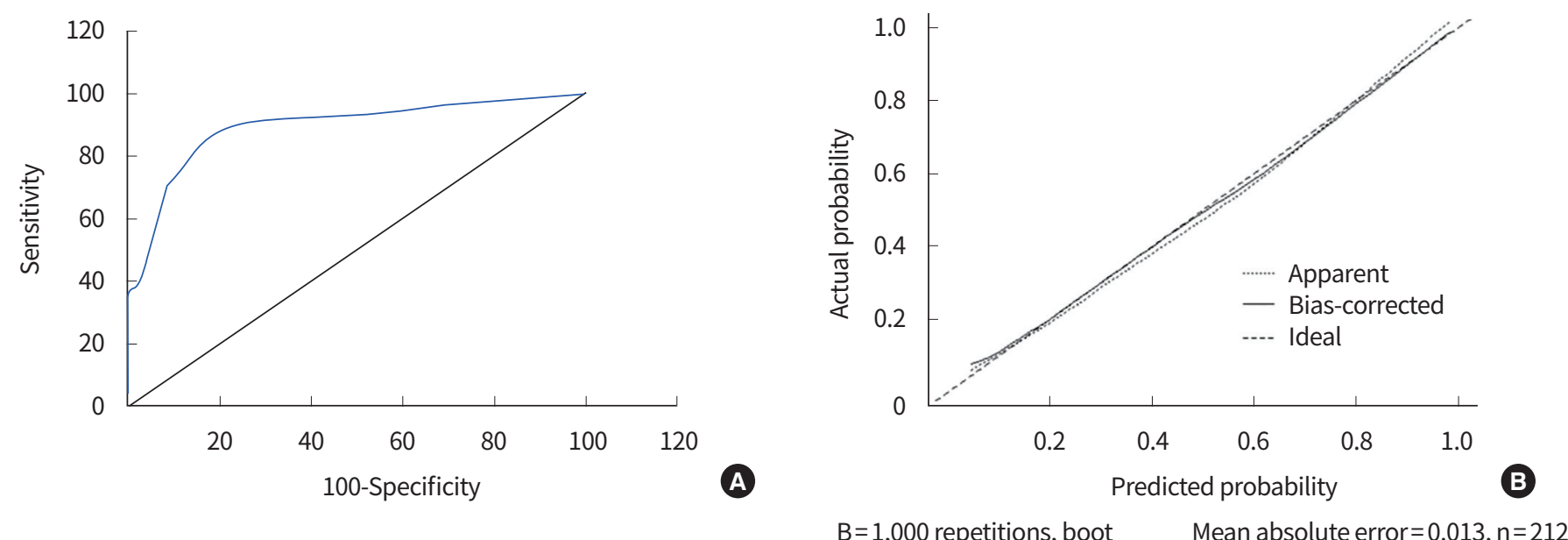

Fig. 7. (A) Receiving operating characteristic (ROC) curve analysis of nomogram. The area under the ROC curve (AUC) value is 0.8915 for the formulated nomogram for the axillary lymph node metastasis. (B) The calibration curve for predicting the axillary lymph node metastasis. The nomogram-predicted axillary lymph node metastasis probabilities are similar to the actual probabilities of axillary lymph node metastasis.

ranged from $60 \%-78 \%$ and $76 \%-97 \%$, respectively [14,17, 18]. Many US studies have yielded results to detect the sensitivity, specificity, and overall accuracy for axillary metastases ranging from $50 \%-92 \%, 90 \%-100 \%$, and $76 \%-92 \%$, respectively [7]. Compared with previous studies using CT or US to predict ALN metastasis, we believe that the performance of our nomogram was relatively sensitive and is comparable as an objective and consistent method.

The application of CT to assess ALN metastasis has been rarely reported, and the results were often inconsistent. Because a normal ALN is seen as a curvilinear-shaped cortex with various axes on $\mathrm{CT}$, unlike the US, it is not easy to measure the smaller and larger diameters of ALNs on CT. According to the pathologic features of ALNs on CT, the peripheral hyperdense cortex is mainly correlated with lymphoid tissue, whereas the central hypodense hilum corresponds to arteries, veins, lymphatic sinuses, and adipose tissue [17]. On CT, the background axillary fat and the fatty hilum of ALNs are not always easily distinguishable because of their low image contrast; however, the hyperdense cortex of ALNs can be evaluated relatively effortlessly and accurately due to the high image contrast. Moreover, cancer cells enter the axillary nodes by the afferent lymphatics and proliferate from the subcapsular sinus of the cortex, extending later through the medulla and finally into the efferent nodal vessels [19]. Metastatic deposits are arrested by these cells in the periphery of a node, causing enlargement of the cortex, which may precede generalized enlargement of the cortex and eventual replacement of the entire node, tending to be lobulated and round [20]. In a CT study of ALN shape by Kutomi et al. [21], it was reported that a round lymph node was an independent predictor of ALN metastasis. These are the reasons why our study focused on CT morphological features of the ALN cortex.

Our study selected variables such as cortical thickness, cortical shape, and strong contrast enhancement as factors to assess ALN metastasis on CT. The current study confirmed that on CT, the cortex of metastatic ALN was significantly thicker than the cortex of non-metastatic ALN, showing morphologically nodular and rounded/irregular changes. Qualitatively assessing the morphological changes in ALNs was better than quantitatively measuring the cortical thickness for predicting ALN metastasis (Table 2). In breast cancer, the primary tumor size is positively correlated with ALN involvement [22-24]. A previous study reported that primary tumor size measured on chest CT correlated with pathologically determined primary tumor size in patients with breast cancer, although most tumor diameters measured on chest $\mathrm{CT}$ and pathology differed by $<5 \mathrm{~mm}$ [25]. Our study adopted the pathologically proven primary tumor size and showed that the primary tumor size was an essential parameter in predicting ALN metastasis (Table 1).

There are several limitations to this study. First, this was a retrospective, single-institution study; therefore, it may even compromise the representativeness of the study. The risk factors may differ when measured by different interpreters due to interobserver variability. Second, there may be a bias due to the difficulty in conducting a paired comparison between CT images and pathological results in each lymph node. Third, external validation is needed to evaluate its predictive ability and generalizability. Fourth, we used the more 
objective pathological T stage of the primary tumor as a measure to predict metastasis, because CT images sometimes tend to overestimate size relative to histopathological criteria [26]. Finally, there may be concerns about the risk of radiation exposure with the use of chest $\mathrm{CT}$ in patients with breast cancer. Although it may still be controversial, the risk of radiation exposure is expected to be relatively insignificant while applying the latest, low-dose $\mathrm{CT}$ techniques in patients with biopsy-proven breast cancer or those planning radiation therapy, and not in cancer screening [27].

In conclusion, the nomogram combining the cortical shape of ALN on preoperative chest CT with primary tumor size showed good performance in predicting ALN metastasis. Thus, chest CT provides reliable predictors of ALN metastasis in breast cancer patients.

\section{CONFLICTS OF INTEREST}

No potential conflict of interest relevant to this article was reported.

\section{ORCID}

Chohee Kim https://orcid.org/0000-0002-3383-8147

Myung Jin Chung https://orcid.org/0000-0002-6271-3343

Semin Chong https://orcid.org/0000-0002-7429-2566

\section{AUTHOR CONTRIBUTIONS}

Conception or design: MJC, SC.

Acquisition, analysis, or interpretation of data: CK, SC.

Drafting the work or revising: $\mathrm{CK}, \mathrm{SC}$.

Final approval of the manuscript: CK, MJC, SC.

\section{REFERENCES}

1. Andersson Y, Bergkvist L, Frisell J, de Boniface J. Longterm breast cancer survival in relation to the metastatic tumor burden in axillary lymph nodes. Breast Cancer Res Treat 2018;171:359-69.

2. Mamounas EP, Kuehn T, Rutgers EJT, von Minckwitz G. Current approach of the axilla in patients with early-stage breast cancer. Lancet 2017 Aug 14 [Epub]. https://doi.org/ 10.1016/S0140-6736(17)31451-4.

3. Li S, Wang X, Yang J, Lv M, Zhang X, Li C, et al. Clinicopathological features and survival of early stage breast cancer in northwest China: a population-based retrospective study of 1287 patients. Thorac Cancer 2018;9:10-8.

4. Gradishar WJ, Anderson BO, Balassanian R, Blair SL, Burstein HJ, Cyr A, et al. NCCN guidelines insights: breast cancer, version 1.2017. J Natl Compr Canc Netw 2017;15: 433-51.

5. Giuliano AE, Ballman K, McCall L, Beitsch P, Whitworth PW, Blumencranz $\mathrm{P}$, et al. Locoregional recurrence after sentinel lymph node dissection with or without axillary dissection in patients with sentinel lymph node metastases: long-term follow-up from the American College of Surgeons Oncology Group (Alliance) ACOSOG Z0011 randomized trial. Ann Surg 2016;264:413-20.

6. Giuliano AE, Hunt KK, Ballman KV, Beitsch PD, Whitworth PW, Blumencranz PW, et al. Axillary dissection vs no axillary dissection in women with invasive breast cancer and sentinel node metastasis: a randomized clinical trial. JAMA 2011;305:569-75.

7. Cools-Lartigue J, Meterissian S. Accuracy of axillary ultrasound in the diagnosis of nodal metastasis in invasive breast cancer: a review. World J Surg 2012;36:46-54.

8. Kamitani T, Hatakenaka M, Yabuuchi H, Matsuo Y, Fujita N, Jinnouchi $\mathrm{M}$, et al. Detection of axillary node metastasis using diffusion-weighted MRI in breast cancer. Clin Imaging 2013;37:56-61.

9. Kim EJ, Kim SH, Kang BJ, Choi BG, Song BJ, Choi JJ. Diagnostic value of breast MRI for predicting metastatic axillary lymph nodes in breast cancer patients: diffusion-weighted MRI and conventional MRI. Magn Reson Imaging 2014;32: 1230-6.

10. Luo N, Su D, Jin G, Liu L, Zhu X, Xie D, et al. Apparent diffusion coefficient ratio between axillary lymph node with primary tumor to detect nodal metastasis in breast cancer patients. J Magn Reson Imaging 2013;38:824-8.

11. Kobayashi H, Kikuchi A, Okazaki S, Ishiguro M, Ishikawa T, lida S, et al. Diagnostic performance of multidetector row computed tomography for assessment of lymph node metastasis in patients with distal rectal cancer. Ann Surg Oncol 2015;22:203-8.

12. Kubota K, Suzuki A, Shiozaki H, Wada T, Kyosaka T, Kishida A. Accuracy of multidetector-row computed tomography in the preoperative diagnosis of lymph node metastasis in patients with gastric cancer. Gastrointest Tumors 2017;3:163-70.

13. Li Y, Diao F, Shi S, Li K, Zhu W, Wu S, et al. Computed tomography and magnetic resonance imaging evaluation of pelvic lymph node metastasis in bladder cancer. Chin J Cancer 2018;37:3. 
14. Shien T, Akashi-Tanaka S, Yoshida M, Hojo T, Iwamoto E, Miyakawa $\mathrm{K}$, et al. Evaluation of axillary status in patients with breast cancer using thin-section CT. Int J Clin Oncol 2008; 13:314-9.

15. Nasu Y, Shikishima H, Miyasaka Y, Nakakubo Y, Ichinokawa $\mathrm{K}$, Kaneko T. A study of the assessment of axillary lymph nodes before surgery for breast cancer using multidetector-row computed tomography. Surg Today 2010;40:10236.

16. Chen CF, Zhang YL, Cai ZL, Sun SM, Lu XF, Lin HY, et al. Predictive value of preoperative multidetector-row computed tomography for axillary lymph nodes metastasis in patients with breast cancer. Front Oncol 2019;8:666.

17. Uematsu T, Sano M, Homma K. In vitro high-resolution helical CT of small axillary lymph nodes in patients with breast cancer: correlation of CT and histology. AJR Am J Roentgenol 2001;176:1069-74.

18. Ogasawara Y, Doihara H, Shiraiwa M, Ishihara S. Multidetector-row computed tomography for the preoperative evaluation of axillary nodal status in patients with breast cancer. Surg Today 2008;38:104-8.

19. Hartveit F, Maehle BO, Halvorsen JF, Tangen M. On the progressive nature of tumour growth in the axillary nodes in breast cancer. Oncology 1983;40:309-14.

20. Bedi DG, Krishnamurthy R, Krishnamurthy S, Edeiken BS, Le-Petross H, Fornage BD, et al. Cortical morphologic features of axillary lymph nodes as a predictor of metastasis in breast cancer: in vitro sonographic study. AJR Am J Roentgenol 2008;191:646-52.

21. Kutomi G, Ohmura T, Satomi F, Takamaru T, Shima H, Su- zuki Y, et al. Lymph node shape in computed tomography imaging as a predictor for axillary lymph node metastasis in patients with breast cancer. Exp Ther Med 2014;8:6815.

22. Meretoja TJ, Leidenius MH, Heikkila PS, Boross G, Sejben I, Regitnig P, et al. International multicenter tool to predict the risk of nonsentinel node metastases in breast cancer. J Natl Cancer Inst 2012;104:1888-96.

23. Ecanow JS, Abe H, Newstead GM, Ecanow DB, Jeske JM. Axillary staging of breast cancer: what the radiologist should know. Radiographics 2013;33:1589-612.

24. Loza CM, Mando P, Ponce C, Colo F, Fabiano V, Loza J, et al. Predictive factors for non-sentinel lymph node metastasis in patients with ACOSOG Z0011 criteria. Breast Care (Basel) 2018;13:434-8.

25. Ahn SJ, Kim YS, Kim EY, Park HK, Cho EK, Kim YK, et al. The value of chest CT for prediction of breast tumor size: comparison with pathology measurement. World J Surg Oncol 2013;11:130.

26. Wienbeck S, Uhlig J, Fischer U, Hellriegel M, von Fintel E, Kulenkampff $D$, et al. Breast lesion size assessment in mastectomy specimens: correlation of cone-beam breast$\mathrm{CT}$, digital breast tomosynthesis and full-field digital mammography with histopathology. Medicine (Baltimore) 2019; 98:e17082.

27. Hagay C, Cherel PJ, de Maulmont CE, Plantet MM, Gilles R, Floiras JL, et al. Contrast-enhanced CT: value for diagnosing local breast cancer recurrence after conservative treatment. Radiology 1996;200:631-8. 